\title{
GLOBAL WELL-POSEDNESS FOR THE GENERALISED FOURTH-ORDER SCHRÖDINGER EQUATION
}

\author{
YUZHAO WANG
}

(Received 2 June 2011)

Abstract

We study the Cauchy problem for the generalised fourth-order Schrödinger equation

$$
i \partial_{t} u+\partial_{x}^{4} u+\partial_{x}\left(|u|^{2 k} u\right)=0, \quad u(0)=u_{0},
$$

for data $u_{0}$ in critical Sobolev spaces $\dot{H}^{1 / 2-3 / 2 k}$. With small initial data we obtain global well-posedness results. Our proof relies heavily on the method developed by Kenig et al. ['Well-posedness and scattering results for the generalised Korteweg-de Vries equation via the contraction principle', Commun. Pure Appl. Math. 46 (1993), 527-620].

2010 Mathematics subject classification: primary 35Q53; secondary 35L30.

Keywords and phrases: fourth-order, Schrödinger equation, global well-posedness.

\section{Introduction}

This paper is mainly concerned with the global well-posedness of the Cauchy problem for the generalised fourth-order Schrödinger equation

$$
\left\{\begin{array}{l}
i \partial_{t} u+\partial_{x}^{4} u+\partial_{x}\left(|u|^{2 k} u\right)=0, \quad k \geq 2,(x, t) \in \mathbb{R} \times \mathbb{R}, \\
u(x, 0)=u_{0}(x),
\end{array}\right.
$$

which occurs in the study of deep water wave dynamics [2], solitary waves [7], vortex filaments [3], and so on. The well-posedness of (1.1) has been widely studied; see [4-6] and references therein.

We show that the initial value problem (1.1) is globally well posed for small data in the critical space $\dot{H}^{s_{k}}$ with $s_{k}=1 / 2-3 / 2 k$, and this allows us to obtain nonlinear scattering in a neighbourhood of the origin in $\dot{H}^{s_{k}}$. This global result is sharp, since $\dot{H}^{s_{k}}$ is the critical space corresponding to (1.1) in the sense that if $u(x, t)$ solves (1.1) with initial data $u_{0}$, then

$$
u_{\lambda}(x, t)=\lambda^{-3 / 2 k} u\left(\frac{x}{\lambda}, \frac{t}{\lambda^{4}}\right), \quad \lambda>0,
$$

(C) 2012 Australian Mathematical Publishing Association Inc. 0004-9727/2012 \$16.00 
solves (1.1) with initial data

$$
u_{0, \lambda}(x)=\lambda^{-3 / 2 k} u_{0}\left(\frac{x}{\lambda}\right), \quad \lambda>0
$$

and such scaling preserves the $\dot{H}^{s_{k}}$ norm, that is, $\left\|u_{0, \lambda}(x)\right\|_{\dot{H}^{s_{k}}}=\left\|u_{0}\right\|_{\dot{H}^{s_{k}}}$.

It is interesting to compare our results with those obtained in [1] for the Schrödinger equation and in [8] for the generalised Korteweg-de Vries (KdV) equation. In [1], Cazenave and Weissler showed that

$$
\left\{\begin{array}{l}
\partial_{t} u=i\left(\partial_{x}^{2} u+|u|^{k} u\right), \quad(x, t) \in \mathbb{R} \times \mathbb{R}, \\
u(x, 0)=u_{0}(x),
\end{array}\right.
$$

is globally well posed in $u_{0} \in \dot{H}^{s_{k}}$ with $s_{k}=1 / 2-2 / k, k \geq 4$, for sufficiently small data. In [8], Kenig, Ponce and Vega showed that

$$
\left\{\begin{array}{l}
\partial_{t} u+\partial_{x}^{3} u+u^{k} u_{x}=0, \quad(x, t) \in \mathbb{R} \times \mathbb{R}, \\
u(x, 0)=u_{0}(x),
\end{array}\right.
$$

is globally well posed in $\dot{H}^{s_{k}}$ with $s_{k}=1 / 2-2 / k, k \geq 4$, for sufficiently small data. Both of these are global well-posedness results in critical spaces; we generalise them to the fourth-order Schrödinger equation.

Our proof relies heavily on the methods developed by Kenig et al. [8], which were concerned with the generalised $\mathrm{KdV}$ equation. We develop some linear estimates for the linear evolution group $W(t)$ by interpolating between the sharp Kato smoothing effect and the maximal function estimate, and employ the Christ-Kiselev lemma to get the inhomogeneous estimates. Then we prove the nonlinear estimates by the Leibniz rule for fractional derivatives [8]. Finally, we get the main theorem by the contraction mapping principle.

1.1. Notation. For $f \in \mathcal{S}^{\prime}$, we denote by $\widehat{f}$ or $\mathcal{F}(f)$ the Fourier transform of $f$. We denote by $\mathcal{F}_{x}$ the Fourier transform on the spatial variable. We write $\omega(\xi)=\xi^{4}$, which is the dispersion relation associated with (1.1). For $u_{0} \in \mathcal{S}^{\prime}(\mathbb{R})$, the linear group $W(t) u_{0}=e^{i t \partial_{x}^{4}} u_{0}$ is defined by

$$
\mathcal{F}_{x}(W(t) \phi)(\xi)=e^{i \xi^{4} t} \hat{\phi}(\xi)
$$

which is the dispersion semigroup associated with (1.1), and it is easy to see that $\|W(t) \phi\|_{L_{x}^{2}}=\|\phi\|_{L_{x}^{2}}$.

Let $1 \leq p, q \leq \infty$. Define

$$
\|f\|_{L_{x}^{p} L_{t}^{q}}=\left(\int_{\mathbb{R}}\left(\int_{\mathbb{R}}|f(x, t)|^{q} d t\right)^{p / q} d x\right)^{1 / p}
$$

and

$$
\|f\|_{L_{t}^{q} L_{x}^{p}}=\left(\int_{\mathbb{R}}\left(\int_{\mathbb{R}}|f(x, t)|^{p} d x\right)^{q / p} d t\right)^{1 / q} .
$$


For $\alpha \in \mathbb{R}$, write

$$
D^{\alpha} f(x, t)=c_{\alpha} \int_{\mathbb{R}} e^{i x \xi}|\xi|^{\alpha} \mathcal{F}_{x}(f)(\xi, t) d \xi
$$

The norm in the Sobolev space $\dot{H}^{s}=D^{-s} L^{2}(\mathbb{R})$ will be denoted by $\|\cdot\|_{\dot{H}^{s}}$, that is, $\left\|v_{0}\right\|_{\dot{H}^{s}}=\left\|D^{s} v_{0}\right\|_{2}$.

1.2. Main results. In this section, we state our main results concerning (1.1) and make some further comments.

Theorem 1.1 (Main theorem). Let $k \geq 2$ and $s_{k}=(k-3) / 2 k$. Then there exists $\delta_{k}>0$ such that, for $u_{0} \in \dot{H}^{s_{k}}$ with

$$
\left\|D^{s_{k}} u_{0}\right\|_{2}<\delta_{k},
$$

there exists a unique strong solution $u$ of (2.1) satisfying

$$
u \in C\left(\mathbb{R} ; \dot{H}^{s_{k}}(\mathbb{R})\right),
$$

and

$$
\left\|D^{S_{k}} u\right\|_{L_{t}^{\infty} L_{x}^{2}}+\left\|D^{S_{k}+1 / 2} u\right\|_{L_{x}^{7} L_{t}^{14 / 3}}+\|u\|_{L_{x}^{14 k / 5} L_{t}^{7 k / 2}}<\infty .
$$

Moreover, the flow map $u_{0} \rightarrow u(t)$ from $\left\{u_{0} \in \dot{H}^{s_{k}}(\mathbb{R}):\left\|u_{0}\right\|_{\dot{H}^{s_{k}(\mathbb{R})<\delta_{k}}}\right\}$ into the class defined by (1.2) and (1.3) is Lipschitz.

The proof will be given in the final section.

REMARK 1.2. The sharpness of this result can be implied by the scaling argument since $\dot{H}^{s_{k}}$ is the critical space under scaling.

REMARK 1.3. By a standard argument (see [8]), we also can prove the small-data global well-posedness in $H^{s}$ with $s \geq s_{k}$, and large-data local well-posedness in $\dot{H}^{s_{k}}$ or $H^{s}$ $\left(s \geq s_{k}\right)$.

\section{Main linear estimates}

In this section we shall deduce all the linear estimates, which are the main tools in the proof of the main theorem stated in the previous section.

2.1. Homogeneous cases. We recall that $W(t)$ denotes the semigroup associated with (1.1), which means that $v(x, t)=W(t) v_{0}$ solves the linear problem associated with (1.1), that is,

$$
\left\{\begin{array}{l}
i \partial_{t} v+\partial_{x}^{4} v=0, \quad(x, t) \in \mathbb{R} \times \mathbb{R} \\
v(x, 0)=v_{0}(x)
\end{array}\right.
$$

First we shall need sharp versions of the smoothing effect of Kato type. 
Lemma 2.1 (Kato smoothing effect). If $u_{0} \in L^{2}$, then

$$
\sup _{x}\left(\int_{\mathbb{R}}\left|D^{3 / 2} W(t) u_{0}(x)\right|^{2} d t\right)^{1 / 2} \leq C\left\|u_{0}\right\|_{2},
$$

where $D^{3 / 2}$ is defined by the Fourier multiplier $|\xi|^{3 / 2}$.

Proof. The estimate (2.2) was given in [9, Theorem 4.1], where it was also shown that this estimate is sharp. Thus we omit the details here.

Now we turn to the maximal function estimate.

LeMma 2.2. If $u_{0} \in \dot{H}^{1 / 4}$, then

$$
\left(\int_{\mathbb{R}}\left(\sup _{t}\left|W(t) u_{0}(x)\right|\right)^{4} d x\right)^{1 / 4} \leq c\left\|u_{0}\right\|_{\dot{H}^{1 / 4}} .
$$

Proof. We see that (2.3) follows from [9, Theorem 2.5].

Before we interpolate between (2.2) and (2.3), we need the following Sobolev embedding.

Lemma 2.3. Let $g \in \mathcal{S}\left(\mathbb{R}^{2}\right)$ and $k \geq 2$. Then

$$
\|g(x, t)\|_{L_{x}^{14 k / 5} L_{t}^{7 k / 2}} \leq c\left\|D^{1 / 4-1 / 2 k} g\right\|_{L_{x}^{28 k /(7 k-4)} L_{t}^{7 k / 2} .}
$$

Proof. We can assume that $k>2$, since the case $k=2$ is trivial. Fix $t$ and use fractional integration in $x$ to obtain the representation

$$
g(x, t)=c \int_{\mathbb{R}} \frac{1}{|x-y|^{1-\alpha_{k}}} D_{x}^{\alpha_{k}} g(y, t) d y,
$$

where $\alpha_{k}=1 / 4-1 / 2 k$. By Minkowski's integral inequality,

$$
\|g(x, t)\|_{L_{t}^{7 k / 2}} \leq c \int_{\mathbb{R}} \frac{1}{|x-y|^{1-\alpha_{k}}}\left\|D_{x}^{\alpha_{k}} g(y, t)\right\|_{L_{t}^{7 k / 2}} d y .
$$

Since

$$
\frac{5}{14 k}=\frac{7 k-4}{28 k}-\left(\frac{1}{4}-\frac{1}{2 k}\right)=\frac{7 k-4}{28 k}-\alpha_{k},
$$

then by fractional integration we obtain the desired result.

Now we give some new estimates implied by (2.2) and (2.3).

LeMma 2.4. (i) If $u_{0} \in L^{2}$, then

$$
\left\|D^{1 / 2} W(t) u_{0}\right\|_{L_{x}^{7} L_{t}^{14 / 3}} \leq c\left\|u_{0}\right\|_{L^{2}} .
$$

(ii) If $u_{0} \in \dot{H}^{1 / 4-1 / k}$, then

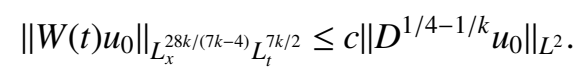


(iii) If $u_{0} \in \dot{H}^{1 / 2-3 / 2 k}$, then

$$
\left\|W(t) u_{0}\right\|_{L_{x}^{14 k / 5} L_{t}^{7 k / 2}} \leq c\left\|D^{1 / 2-3 / 2 k} u_{0}\right\|_{L^{2}} .
$$

Proof. Part (i) follows from interpolating between (2.2) and (2.3), since

$$
\frac{1}{7}=\frac{\theta}{\infty}+\frac{1-\theta}{4}, \quad \frac{3}{14}=\frac{\theta}{2}+\frac{1-\theta}{\infty}, \quad \frac{1}{2}=\frac{3 \theta}{2}-\frac{1-\theta}{4}
$$

with $\theta=3 / 7$.

For (ii), since

$$
\frac{7 k-4}{28 k}=\frac{\theta}{\infty}+\frac{1-\theta}{4}, \quad \frac{2}{7 k}=\frac{\theta}{2}+\frac{1-\theta}{\infty}, \quad \frac{1}{4}-\frac{1}{k}=-\frac{3 \theta}{2}+\frac{1-\theta}{4},
$$

with $\theta=4 / 7 k$, the result follows from a similar interpolation argument.

Part (iii) follows directly from (2.4) and (2.6).

2.2. Inhomogeneous cases. The main tool in this section is the following ChristKiselev lemma given by Molinet and Ribaud [10].

Lemma 2.5. Let $T$ be a linear operator defined on space-time function $f(x, t)$ by

$$
T f(t)=\int_{\mathbb{R}} K\left(t, t^{\prime}\right) f\left(t^{\prime}\right) d t^{\prime},
$$

such that

$$
\|T f\|_{L_{x}^{p_{1}} L_{t}^{q_{1}}} \leq C\|f\|_{L_{x}^{p_{2}} L_{t}^{q_{2}}}
$$

where $\min \left(p_{1}, q_{1}\right)>\max \left(p_{2}, q_{2}\right)$. Then

$$
\left\|\int_{0}^{t} K\left(t, t^{\prime}\right) f\left(t^{\prime}\right) d t^{\prime}\right\|_{L_{x}^{p_{1}} L_{t}^{q_{1}}} \leq C\|f\|_{L_{x}^{p_{2}} L_{t}^{q_{2}}} .
$$

Now we begin to state our main estimates in this section.

Lemma 2.6. If $g \in L_{x}^{7 / 6} L_{t}^{14 / 11}$, then

$$
\begin{gathered}
\left\|\partial_{x} \int_{0}^{t} W(t-s) g(s) d s\right\|_{L_{x}^{7} L_{t}^{14 / 3}} \leq c\|g\|_{L_{x}^{7 / 6} L_{t}^{14 / 11}}, \\
\left\|D_{x}^{1 / 2} \int_{0}^{t} W(t-s) g(s) d s\right\|_{L_{t}^{\infty} L_{x}^{2}} \leq c\|g\|_{L_{x}^{7 / 6} L_{t}^{14 / 11}} \\
\left\|D^{3 / 2 k} \int_{0}^{t} W(t-s) g(s) d s\right\|_{L_{x}^{(14 k) / 5} L_{t}^{(7 k) / 2}} \leq c\|g\|_{L_{x}^{7 / 6} L_{t}^{14 / 11} .}
\end{gathered}
$$

Proof. (i) The dual of (2.5) is

$$
\left\|D^{1 / 2} \int_{\mathbb{R}} W(-s) g(s) d s\right\|_{L_{x}^{2}} \leq c\|g\|_{L_{x}^{7 / 6} L_{t}^{14 / 11}}
$$


so by $(2.5)$,

$$
\left\|\partial_{x} \int_{\mathbb{R}} W(t-s) g(s) d s\right\|_{L_{x}^{7} L_{t}^{14 / 3}} \leq c\left\|D^{1 / 2} \int_{\mathbb{R}} W(-s) g(s) d s\right\|_{L_{x}^{2}},
$$

which can be bounded by

$$
c\|g\|_{L_{x}^{7 / 6} L_{t}^{14 / 11}}
$$

in view of (2.8). Thus we can complete the proof by the Christ-Kiselev lemma 2.5.

(ii) In view of the unitary property of the semigroup $W(t)$, it is easy to see that

$$
\left\|D^{1 / 2} \int_{\mathbb{R}} W(t-s) g(s) d s\right\|_{L_{t}^{\infty} L_{x}^{2}} \leq\left\|D^{1 / 2} \int_{\mathbb{R}} W(-s) g(s) d s\right\|_{L_{x}^{2}} .
$$

Then the rest of the proof is the same as the previous one.

(iii) By (2.7) and (2.8) we have

$$
\begin{aligned}
& \| D^{3 / 2 k} \int_{\mathbb{R}} W(t-s) g(s) d s \|_{L_{x}^{14 k / 5} L_{t}^{7 k / 2}} \\
& \leq c\left\|D^{1 / 2} \int_{\mathbb{R}} W(-s) g(s) d s\right\|_{L_{x}^{2}} \leq c\|g\|_{L_{x}^{7 / 6} L_{t}^{14 / 11} .}
\end{aligned}
$$

Then we complete the proof by applying Lemma 2.5 .

\section{Nonlinear estimates}

We shall need the following nonlinear estimate in the next section.

LeMma 3.1. If $s_{k}=1 / 2-3 / 2 k$ with $k \geq 2$, then

$$
\left\|D_{x}^{s_{k}+1 / 2}\left(|v|^{2 k} v\right)\right\|_{L_{x}^{7 / 6} L_{t}^{14 / 11}} \leq c\left\|D_{x}^{s_{k}+1 / 2} v\right\|_{L_{x}^{7} L_{t}^{14 / 3}}\|v\|_{L_{x}^{14 k / 5} L_{t}^{7 k / 2}}^{2 k} .
$$

The proof of this nonlinear estimate is based on the following chain rule and Leibniz rule for fractional derivatives (see [9]).

Lemma 3.2 (Chain rule for fractional derivatives). Let $\alpha \in(0,1)$ and $p, q, p_{1} \in(1, \infty)$, $q_{1} \in(1, \infty]$, such that

$$
\frac{1}{p}=\frac{1}{p_{1}}+\frac{1}{p_{2}} \quad \text { and } \quad \frac{1}{q}=\frac{1}{q_{1}}+\frac{1}{q_{2}} .
$$

Then

$$
\left\|D^{\alpha} F(f)\right\|_{L_{x}^{p} L_{T}^{q}} \leq c\left\|F^{\prime}(f)\right\|_{L_{x}^{p_{1}} L_{T}^{q_{1}}}\left\|D^{\alpha} f\right\|_{L_{x}^{p_{2}} L_{T}^{q_{2}}}
$$

Lemma 3.3 (Leibniz rule for fractional derivatives). Let $\alpha \in(0,1)$ and let $p, q, p_{1}, q_{1}, p_{2}, q_{2} \in(1, \infty)$ be such that

$$
\frac{1}{p}=\frac{1}{p_{1}}+\frac{1}{p_{2}} \text { and } \quad \frac{1}{q}=\frac{1}{q_{1}}+\frac{1}{q_{2}} .
$$

Then

$$
\left\|D^{\alpha}(f g)-f D^{\alpha} g\right\|_{L_{x}^{p} L_{T}^{q}} \leq c\left\|D^{\alpha} f\right\|_{L_{x}^{p_{1}} L_{T}^{q_{1}}}\|g\|_{L_{x}^{p_{2}} L_{T}^{q_{2}}}
$$


Proof of Lemma 3.1. Let $s=s_{k}-1 / 2$. We first apply Lemma 3.3 with $f=v^{k+1}$ and $g=\bar{v}^{k}$, to give

$$
\left\|D^{s}\left(|v|^{2 k} v\right)-v^{k+1} D^{s}\left(\bar{v}^{k}\right)\right\|_{L_{x}^{7 / 6} L_{t}^{14 / 11}} \leq c\left\|D^{s}\left(v^{k+1}\right)\right\|_{L_{x}^{2} L_{t}^{2}}\left\|\bar{v}^{k}\right\|_{L_{x}^{14 / 5} L_{t}^{7 / 2}} .
$$

Then by Lemma 3.2, the above can be bounded by

$$
c\left\|D^{s} v\right\|_{L_{x}^{7} L_{t}^{14 / 3}}\left\|v^{k}\right\|_{L_{x}^{14 / 5} L_{t}^{7 / 2} \| \bar{v}^{k}}\left\|_{L_{x}^{14 / 5} L_{t}^{7 / 2}} \leq c\right\| D_{x}^{S_{k}+1 / 2} v\left\|_{L_{x}^{7} L_{t}^{14 / 3}}\right\| v \|_{L_{x}^{14 k / 5} L_{t}^{7 k / 2}}^{2 k}
$$

In order to complete the proof, we need to bound $\left\|v^{k+1} D^{s}\left(\bar{v}^{k}\right)\right\|_{L_{x}^{7 / 6} L_{t}^{14 / 11}}$. By Hölder's inequality, it can be bounded by

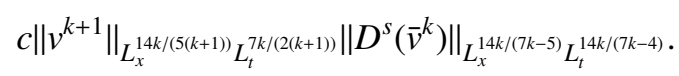

Then by Lemma 3.2, the above can be bounded by

$$
\begin{aligned}
& c\left\|v^{k+1}\right\|_{L_{x}^{14 k /(5(k+1))} L_{t}^{7 k /(2(k+1))}}\left\|D^{S} \bar{v}\right\|_{L_{x}^{7} L_{t}^{14 / 3}}\left\|v^{k-1}\right\|_{L_{x}^{14 k /(5(k-1))} L^{7 k /(2(k-1))}} \\
& \quad \leq c\left\|D_{x}^{s_{k}+1 / 2} v\right\|_{L_{x}^{7} L_{t}^{14 / 3}}\|v\|_{L_{x}^{14 k / 5} L_{t}^{7 k / 2} .}
\end{aligned}
$$

Thus we get the nonlinear estimate (3.1).

\section{Proof of main theorem}

In this section we shall prove our main result, that is, that the initial value problem (1.1) is globally well posed in a neighbourhood of the origin in $\dot{H}^{s_{k}}$ with $k \geq 2$.

Proof of Theorem 1.1. For $\omega: \mathbb{R}^{2} \rightarrow \mathbb{R}$ define

$$
\begin{aligned}
& \eta_{1}(\omega)=\left\|D_{x}^{s_{k}} \omega\right\|_{L_{t}^{\infty} L_{x}^{2}}, \\
& \eta_{2}(\omega)=\left\|D_{x}^{s_{k}+1 / 2} \omega\right\|_{L_{x}^{7} L_{t}^{14 / 3},}, \\
& \eta_{3}(\omega)=\|\omega\|_{L_{x}^{14 k / 5} L_{t}^{7 k / 2},}^{3} \\
& \Omega(\omega)=\sum_{i=1}^{3} \eta_{i}(\omega),
\end{aligned}
$$

and

$$
X=\left\{\omega \in C\left(\mathbb{R} ; L^{2}(\mathbb{R})\right): \Omega(\omega)<\infty\right\} .
$$

In view of Lemma 2.4 and the unitary property of the group $W(t)$, it is easy to see that

$$
\Omega\left(W(t) u_{0}\right) \leq c\left\|D^{s_{k}} u_{0}\right\|_{2} .
$$

For $u_{0} \in L^{2}(\mathbb{R})$ and $v \in X$, we denote by $u=\Phi(v)=\Phi_{u_{0}}(v)$ the solution of the linear problem

$$
\left\{\begin{array}{l}
i \partial_{t} u+\partial_{x}^{4} u+\partial_{x}\left(|v|^{2 k} v\right)=0, \quad k \geq 2,(x, t) \in \mathbb{R} \times \mathbb{R} \\
u(x, 0)=u_{0}(x)
\end{array}\right.
$$


which has an equivalent integral form,

$$
u(t)=W(t) u_{0}-\int_{0}^{t} W(t-s) \partial_{x}\left(|v|^{2 k} v\right)(s) d s .
$$

We shall prove that $\Phi$ is a contraction map in a neighbourhood of the origin in $\dot{H}^{s_{k}}$, that is, there exist $\delta>0$ and $a>0$ such that if $\left\|u_{0}\right\|_{\dot{H}^{s_{k}}}$ then

$$
\Phi: X_{a} \rightarrow X_{a}
$$

is a contraction map, where

$$
X_{a}=\{\omega \in X: \Omega(\omega) \leq a\}
$$

We shall use the integral equation (4.1). Inserting the linear estimates from Lemmas 2.4 and 2.6 into (4.1) and then using Lemma 3.1,

$$
\begin{aligned}
\eta_{j}(\Phi(v)) & \leq c\left\|D^{s_{k}} u_{0}\right\|_{2}+c\left\|D_{x}^{s_{k}+1 / 2}\left(|v|^{2 k} v\right)\right\|_{L_{x}^{7 / 6} L_{t}^{14 / 11}} \\
& \leq c\left\|D^{s_{k}} u_{0}\right\|_{2}+c \eta_{2}(v) \eta_{3}^{2 k}(v),
\end{aligned}
$$

for $j=1,2,3$ and $v \in X$. Thus we have

$$
\Omega(\Phi(v)) \leq c\left\|u_{0}\right\|_{2}+c(\Omega(v))^{2 k+1} .
$$

If we choose $\delta$ such that

$$
c(4 c \delta)^{2 k}=\frac{1}{2}
$$

and

$$
a \in(2 c \delta, 3 c \delta)
$$

then

$$
\Phi: X_{a} \rightarrow X_{a}
$$

A similar argument shows that

$$
\begin{aligned}
\Omega\left(\Phi(v)-\Phi\left(v^{\prime}\right)\right) & \leq c\left[\Omega(\Phi(v))+\Omega\left(\Phi\left(v^{\prime}\right)\right)\right]^{2 k} \Omega\left(v-v^{\prime}\right) \\
& \leq 2 c a^{2 k} \Omega\left(v-v^{\prime}\right)<\frac{1}{2} \Omega\left(v-v^{\prime}\right) .
\end{aligned}
$$

Thus we can complete the proof by the standard contraction argument (as in [8]).

\section{Acknowledgement}

We are grateful to the reviewers for careful reading and comments. 


\section{References}

[1] T. Cazenave and F. B. Weissler, 'The Cauchy problem for the critical nonlinear Schrödinger equation in $H^{s}$, Nonlinear Analysis TMA 14 (1990), 807-836.

[2] K. B. Dysthe, 'Note on a modification to the nonlinear Schrödinger equation for application to deep water waves', Proc. R. Soc. Lond. Ser. A 369 (1979), 105-114.

[3] Y. Fukumoto, 'Motion of a curved vortex filament: higher-order asymptotics', Proc. IUTAM Symp. Geom. Stat. Turbul. (2001), 211-216.

[4] C. Guo and S. Cui, 'Global existence of solutions for a fourth-order nonlinear Schrödinger equation', Appl. Math. Lett. 19 (2006), 706-711.

[5] Z. Huo and Y. Jia, 'The Cauchy problem for the fourth-order nonlinear Schrödinger equation related to the vortex filament', J. Differential Equations 214 (2005), 1-35.

[6] C. Hao, L. Hsian and B. Wang, 'Wellposedness for the fourth order nonlinear Schrödinger equations', J. Math. Anal. Appl. 320 (2006), 246-265.

[7] V. I. Karpman, 'Stabilization of soliton instabilities by higher-order dispersion: fourth-order nonlinear Schrödinger-type equations', Phys. Rev. E 53 (1996), 1336-1339.

[8] C. Kenig, G. Ponce and L. Vega, 'Well-posedness and scattering results for the generalised Korteweg-de Vries equation via the contraction principle', Commun. Pure Appl. Math. 46 (1993), 527-620.

[9] C. E. Kenig, G. Ponce and L. Vega, 'Oscillatory integrals and regularity of dispersive equations', Indiana Univ. Math. J. 40 (1991), 33-69.

[10] L. Molinet and F. Ribaud, 'Well-posedness results for the generalised Benjamin-Ono equation with small initial data', J. Math. Pures Appl. 83 (2004), 277-311.

YUZHAO WANG, Department of Mathematics and Physics,

North China Electric Power University, Beijing 102206, PR China

e-mail:wangyuzhao2008@gmail.com 